\title{
A Case of Solitary Plasmacytoma of Bone Showing Coexpression of Both Immunoglobulin Light Chains
}

\section{Ryota Matsuoka}

University of Tsukuba https://orcid.org/0000-0002-8887-1593

Noriaki Sakamoto ( $\nabla$ n.sakamoto@md.tsukuba.ac.jp )

University of Tsukuba https://orcid.org/0000-0001-6573-6463

\section{Takayasu Kato}

University of Tsukuba

\section{Shigeru Chiba}

University of Tsukuba

Masayuki Noguchi

University of Tsukuba

\section{Research Article}

Keywords: solitary plasmacytoma of bone, dual immunoglobulin light chain expression, allelic exclusion, isotypic exclusion, case report

Posted Date: November 12th, 2021

DOI: https://doi.org/10.21203/rs.3.rs-1053107/v1

License: (c) (i) This work is licensed under a Creative Commons Attribution 4.0 International License.

Read Full License 


\section{Abstract}

Background: Solitary plasmacytoma of bone (SPB) is a rare plasma cell neoplasm. It arises in bone as a single locus in the absence of any plasma cell myeloma lesions. Plasma cell neoplasms intrinsically express only one immunoglobulin light chain ( $\mathrm{g} \mathrm{L} \mathrm{L})$ - kappa or lambda - and using this fact, kappa/lambda deviation is the decisive factor for diagnosis. Coexpression of both IgLs in a single tumor cell is extremely rare.

Case presentation: We report a case of SPB that arose in the vertebra of a 52-year-old Japanese woman. Histologically, the resected mass showed diffuse plasma cell proliferation. Dual IgL expression was detected by flow cytometry, immunohistochemistry and in situ hybridization (ISH) targeting IgL mRNA.

Conclusion: We have presented an extremely rare case of SPB showing dual expression of kappa and lambda IgLs. This unusual case of plasma cell neoplasia might represent a possible exceptional example of failure of "IgL isotypic exclusion".

\section{Background}

Solitary plasmacytoma of bone (SPB) is a rare subtype of plasma cell neoplasm, accounting for less than $5 \%$ of plasma cell neoplasms overall. SPB may develop at any bone site, but is especially associated with active bone marrow hematopoiesis. Reported sites of origin have included the vertebrae, ribs and pelvis. ${ }^{1,2}$ Cytologically and immunophenotypically, SPB is similar to plasma cell myeloma (PCM), although it presents as a single localized neoplasm, shows no evidence of bone marrow involvement, and no clinical or laboratory evidence of PCM is evident in affected patients. The median age of patients with SPB is 55 years, i.e. about 10 years younger than those with PCM, and males are affected twice as frequently as females. SPB is treated mainly with radiation and surgery, with additional chemotherapy in cases that have progressed to PCM.

The prognosis of SPB is better than that of PCM, and 50\% of SPB patients survive longer than 10 years. More than $50 \%$ of SPB cases progress to PCM within 2 years or less, suggesting that some cases of SBP represent an early manifestation of PCM. ${ }^{1,3-5}$

Normal B-cells or plasma cells express a single immunoglobulin kappa or lambda light chain, and the ratio of B-cells or plasma cells expressing kappa relative to those expressing lambda ranges from 0.5 to 3.0. ${ }^{6,7}$ B-cell and plasma cell neoplasms are believed to arise from a single cell after initiation of immunoglobulin heavy chain $(\mathrm{lgH})$ and immunoglobulin light chain $(\mathrm{lgL})$ rearrangement, and the neoplastic plasma cells essentially express a single type of IgL. Thus, restriction of IgL expression can be detected by flow cytometry, in situ hybridization and immunohistochemistry. In neoplastic plasma cells, co-expression of both IgL types is extremely rare. Here, we report a very rare case of SPB showing dual expression of IgL. 


\section{Case Presentation}

A 36-year-old woman presented to a local hospital with a history of neck pain. Computed tomography (CT) and magnetic resonance imaging (MRI) demonstrated a tumor arising from the anterior elements of the $\mathrm{C} 1$ and $\mathrm{C} 2$ vertebrae. Cervical spine fusion and mass reduction surgery were performed. The resected specimen showed diffuse proliferation of plasma cells and was diagnosed as "plasmacytoma" at that time. Additional radiotherapy was performed, but the patient later dropped out from the treatment course.

Sixteen years later, at the age of 52 years, the patient returned complaining of dysarthria. CT and MRI showed a similar but much larger mass at the same location, and recurrence of the tumor was diagnosed (Figure 1). The mass compressed the spinal cord and was thought to be responsible for the dysarthria. The patient was referred to our hospital for further examination and treatment. Quantitative serum immunoglobulin (lg) analysis showed an increased level of lgG $(2096 \mathrm{mg} / \mathrm{dl}$; reference range 870-1700 $\mathrm{mg} / \mathrm{dl})$ and normal levels of $\operatorname{lgM}(203 \mathrm{mg} / \mathrm{dl}$; reference range $46-260 \mathrm{mg} / \mathrm{dl})$ and $\operatorname{lgA}(293 \mathrm{mg} / \mathrm{dl}$; reference range $110-410 \mathrm{mg} / \mathrm{dl})$. A serum Ig free light chain study revealed increased levels of both free kappa light chain $(61.5 \mathrm{mg} / \mathrm{l}$; reference range $2.42-18.92 \mathrm{mg} / \mathrm{l})$ and free lambda light chain $(88.1 \mathrm{mg} / \mathrm{l}$; reference range 4.44-26.18 mg/l) (kappa/lambda: 0.70).

Excisional biopsy of the tumor was performed. Flow cytometry analysis demonstrated a distinct population of plasma cells expressing CD38 and co-expression of cytoplasmic kappa and lambda light chain (Figure 2). Histologically, the tumor specimen showed diffuse plasma cell proliferation, and immunohistochemistry showed that these plasma cells were positive for CD138 (Figure 3a, b) and MUM1, and negative for CD3, CD20 and CD56. Immunohistochemistry for IgL and in situ hybridization (ISH) targeting IgL mRNA demonstrated co-expression of kappa and lambda light chain (Figure 3d-g). Bone marrow biopsy showed no evidence of plasmacytes showing dual expression or deviation of kappa and lambda light chain., and additional CT and MRI revealed no skeletal abnormality except for the primary lesion, and absence of end-organ damage attributable to a proliferative plasma cell disorder.

We reviewed the previous specimen and performed additional IgL immunohistochemistry. The sample revealed a histological pattern similar to that of the later sample as well as co-expression of kappa and lambda light chain (Supplementary figure 1).

Taking these findings together, the tumor was diagnosed as SPB with dual expression of the kappa and lambda light chains. After diagnosis, the patient underwent nine courses of VRD (bortezomib, lenalidomide and dexamethasone) therapy, which reduced the size of the lesion. Two years after diagnosis, the lesions have not increased in size, and bone marrow examinations have shown no progression to PCM.

\section{Discussion}

To our knowledge, this case of SPB showing dual expression of the kappa and lambda light chains is the first of its kind to have been reported. Normal plasma cells differentiate from B-cells and produce Ig. B- 
cells can rearrange their Ig genes to recognize a huge variety of different antigens. Ig is normally composed of one heavy and one light chain. There are five types of $\lg \mathrm{H}-\lg \mathrm{M}, \lg \mathrm{G}, \lg \mathrm{A}, \lg \mathrm{D}$ and $\lg \mathrm{E}$ produced by a single gene - and two types of IgL - kappa and lambda produced by two distinct genes. The $I g H$, IgL kappa, and IgL lambda gene loci are located on chromosome 14,2 , and 22, respectively. If the $I g H$ and one of the $I g L$ genes are rearranged successfully, the other allele of the lg gene is excluded to maintain the antigen specificity of the B-cell, a phenomenon known as "allelic exclusion". In this way, a single B-cell expresses the rearranged $l g H$ and the either $l g L$ genes transcribed from only one allele each, with the other 4 alleles remaining in the germline configuration. Normal B-cells first undergo $\operatorname{lgH}$ rearrangement, followed by $\lg L$ kappa rearrangement. If a productive $\lg L$ kappa rearrangement occurs, the IgL lambda gene never rearranges. If IgL kappa rearrangement is nonproductive for both alleles, the IgL kappa locus is inactivated by deletion and I $g L$ lambda rearrangement occurs. ${ }^{8,9}$ This mechanism of expressing either IgL kappa or lambda genes is called "isotypic exclusion", and thus B-cells (or plasma cells) express one type of light chain, but not both. ${ }^{10}$ "Isotypic exclusion" of IgL is not fully understood, although some hypotheses have been proposed on the basis of experimental studies. Inactivation of IgL kappa is accomplished by recombination activating gene (RAG)-mediated joining of the non-coding recombining sequence (RS). The RS is the IgL kappa-deleting element in humans, located $\sim 25$ kilobases downstream of the constant region of $I g L$ kappa (C-kappa). ${ }^{11,12} \mathrm{RS}$ recombination leads to deletion of the C-kappa exon and silencing of the IgL kappa allele, and it is also known that RS recombination promotes the formation of B cells which express IgL lambda chain. ${ }^{12-14}$ However, Diaw et al. reported a mouseorigin plasmacytoma that produced both IgL kappa and lambda, and using micro-manipulation and RTPCR confirmed that both were expressed simultaneously in a single cell. ${ }^{18}$ This suggests that in some plasma cell neoplasms both kappa and lambda light chains may exist in a single plasma cell. Furthermore, few cases of PCM showing dual expression of IgL have been reported. The majority of such cases have tended to show a high incidence of complex cytogenetic or FISH abnormalities, suggesting involvement of the light chain genes, subsequent isotypic exclusion error, and dual light chain expression. ${ }^{15-17}$ Unfortunately, as no investigation of chromosome abnormalities was undertaken in the present case, any genetic dysfunction related to isotypic exclusion remained unclear. On the other hand, Shi et al. subjected human peripheral B-cells to single-cell sequencing and found that more than one antibody was produced in some individual B-cells, albeit accounting for a small proportion of the total (about $10 \%$ ). ${ }^{19}$ This unprecedented finding appears to cast doubt on the traditional "one cell - one antibody" paradigm and suggested that dual expression of kappa and lambda light chain can occur in normal conditions and might be the origin of the dual IgL expression neoplasm. However, due to the limited number of cases, further investigation is needed to elucidate the mechanism of tumor coexpression of kappa and lambda light chains.

The findings in the present case invite speculation as to how both the kappa and lambda light chains can exist in a single neoplastic plasma cell. Two hypotheses to explain this have been suggested: (1) two types of light chain are expressed in the same antibody, (2) two types of antibodies constructed by each of the kappa and lambda light chains are present in the same plasma cell. In the present case, we detected coexpression of both IgLs in the neoplastic cells by flow cytometry, immunohistochemistry and 
in situ hybridization (ISH) targeting IgL mRNA, but none of the results offered any suggestion about either hypothesis. There is no way to test these hypothesis at this stage, and further analysis is needed.

\section{Conclusion}

To our knowledge, the present case of SPB showing dual IgL expression is the first of its kind to have been reported. IgL rearrangement is under strict genetic control, although the mechanism involved is still unclear. The present appears to represent an exceptional event that deviates from the traditional "isotypic exclusion" mechanism.

\section{Abbreviations}

SPB: solitary plasmacytoma of bone

PCM: plasma cell myeloma

IgL: immunoglobulin light chain

IgH: immunoglobulin heavy chain

CT: computed tomography

MRI: magnetic resonance imaging

VRD: bortezomib, lenalidomide and dexamethasone

Ig: immunoglobulin

\section{Declarations}

Ethics approval and consent to participate

Not applicable.

Consent for publication

The patient has given consent for the case report.

Availability of data and materials

All data and material are included in this published article.

Competing interests

The authors have no conflicts to disclose. 
Funding

Not applicable.

Author information

1) Affiliations

Department of Diagnostic Pathology, Faculty of Medicine, University of Tsukuba, 1-1-1 Tennodai, Tsukuba, Ibaraki 305-8577 Japan

Ryota Matsuoka, Noriaki Sakamoto, Masayuki Noguchi

Department of Hematology, Faculty of Medicine, University of Tsukuba, 1-1-1 Tennodai, Tsukuba, Ibaraki 305-8577 Japan

Takayasu Kato, Shigeru Chiba

2) Authors' contributions

All authors contributed to the writing of the manuscript and approved the final manuscript.

Acknowledgements

Not applicable.

\section{References}

1. Dimopoulos, M.A., Moulopoulos, L.A., Maniatis, A. \& Alexanian, R. Solitary plasmacytoma of bone and asymptomatic multiple myeloma. Blood 96, 2037-2044 (2000).

2. Kumar, P. et al. Solitary plasmacytoma of the proximal tibia in an adolescent. Pediatr. Blood Cancer 56, 158-60 (2011).

3. de Waal, E.G.M. et al. Progression of a solitary plasmacytoma to multiple myeloma. A populationbased registry of the northern Netherlands. Br. J. Haematol. 175, 661-667 (2016).

4. Hill, Q.A., Rawstron, A.C., De Tute, R.M. \& Owen, R.G. Outcome prediction in plasmacytoma of bone: A risk model utilizing bone marrow flow cytometry and light-chain analysis. Blood 124, 1296-1299 (2014).

5. Knobel, D. et al. Prognostic factors in solitary plasmacytoma of the bone: A multicenter Rare Cancer Network study. BMC Cancer 6, 1-9 (2006).

6. Kroft, S.H. Monoclones, monotypes, and neoplasia: Pitfalls in lymphoma diagnosis. Am. J. Clin. Pathol. 121, 457-459 (2004).

7. Hristov, A.C., Comfere, N.I., Vidal, C.I. \& Sundram, U. Kappa and lambda immunohistochemistry and in situ hybridization in the evaluation of atypical cutaneous lymphoid infiltrates. J. Cutan. Pathol. 47, 
1103-1110 (2020).

8. Moore, M.W., Durdik, J., Persiani, D.M. \& Selsing, E. Deletions of $\mathrm{k}$ chain constant region genes in mouse $\lambda$ chain-producing $B$ cells involve intrachromosomal DNA recombinations similar to $V-J$ joining. Proc. Natl. Acad. Sci. U.S.A. 82, 6211-6215 (1985).

9. Retter, M.W. \& Nemazee, D. Receptor editing occurs frequently during normal B cell development. J. Exp. Med. 188, 1231-1238 (1998).

10. Xu, D. Dual surface immunoglobulin light-chain expression in B-cell lymphoproliferative disorders. Arch. Pathol. Lab. Med. 130, 853-856 (2006).

11. Siminovitch, K.A., Moore, M.W., Durdik, J. \& Selsing, E. The human kappa deleting element and the mouse recombining segment share DNA sequence homology. Nucleic Acids Res. 15, 2699-2705 (1987).

12. Vettermann, C. \& Schlissel, M.S. Allelic exclusion of immunoglobulin genes: Models and mechanisms. Immunol. Rev. 237, 22-42 (2010).

13. Nemazee, D. Receptor editing in lymphocyte development and central tolerance. Nat. Rev. Immunol. 6, 728-740 (2006).

14. Vela, J. L., Aït-Azzouzene, D., Duong, B.H., Ota, T. \& Nemazee, D. Rearrangement of mouse immunoglobulin kappa deleting element recombining sequence promotes immune tolerance and lambda B cell production. Immunity 28, 161-70 (2008).

15. Jiang, A.S. et al. Plasma cell myeloma with dual expression of kappa and lambda light chains. Int. J. Clin. Exp. Pathol. 11, 4718-4723 (2018).

16. Gentry, M., Pettenati, M. \& Pang, C.S. Biclonal light chain gammopathy with aberrant CD33 expression in secondary plasma cell leukemia. Int. J. Clin. Exp. Pathol. 6, 2224-2229 (2013).

17. Jiwani, S., Bornhost, J. \& Alapat, D. Biphenotypic plasma cell myeloma: Two cases of plasma cell neoplasm with a coexpression of kappa and lambda light chains. Int. J. Clin. Exp. Pathol. 8, 85368544 (2015).

18. Diaw, L., Siwarski, D., DuBois, W., Jones, G. \& Huppi, K. Double producers of kappa and lambda define a subset of B cells in mouse plasmacytomas. Mol. Immunol. 37, 775-781 (2000).

19. Shi, Z. et al. More than one antibody of individual B cells revealed by single-cell immune profiling. Cell Discov. 5, (2019).

\section{Figures}



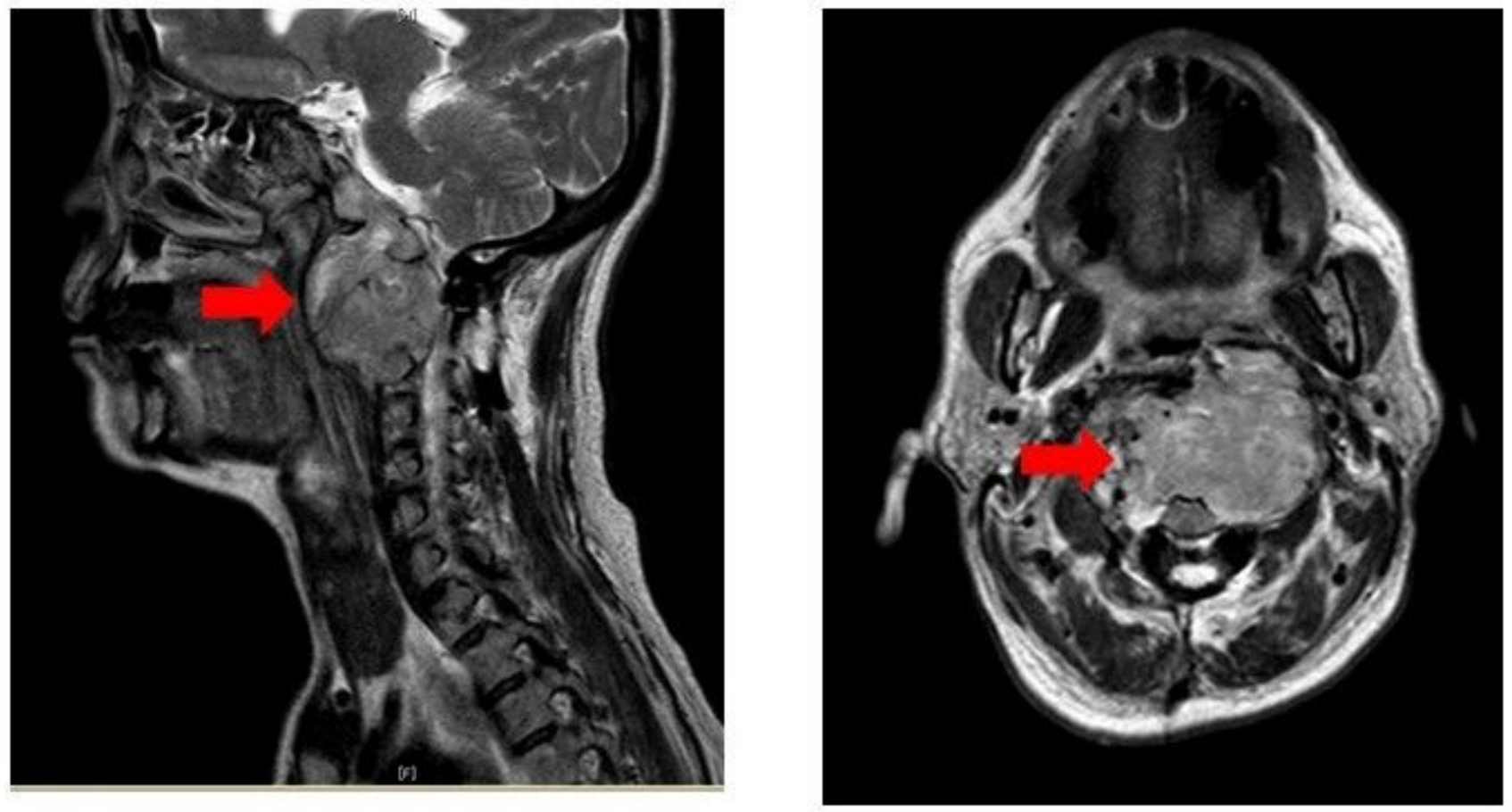

Figure 1

T2-weighted MRI revealed a hyper-intensity signal mass lesion extending from the clivus to the atlas vertebra (red arrow).
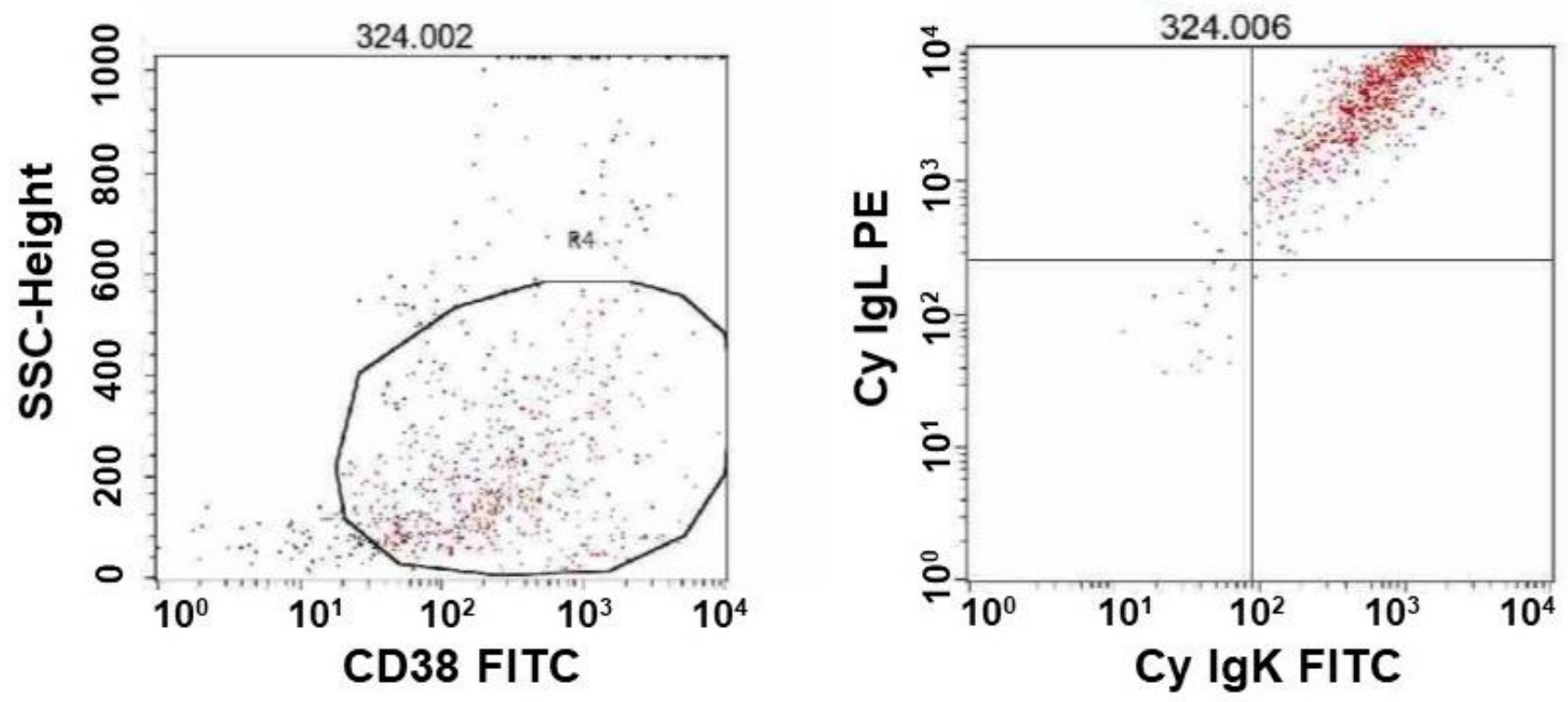

Figure 2 
Flow cytometry of the tumor revealed that CD38-positiev cells co-expressed kappa and lambda light chain.
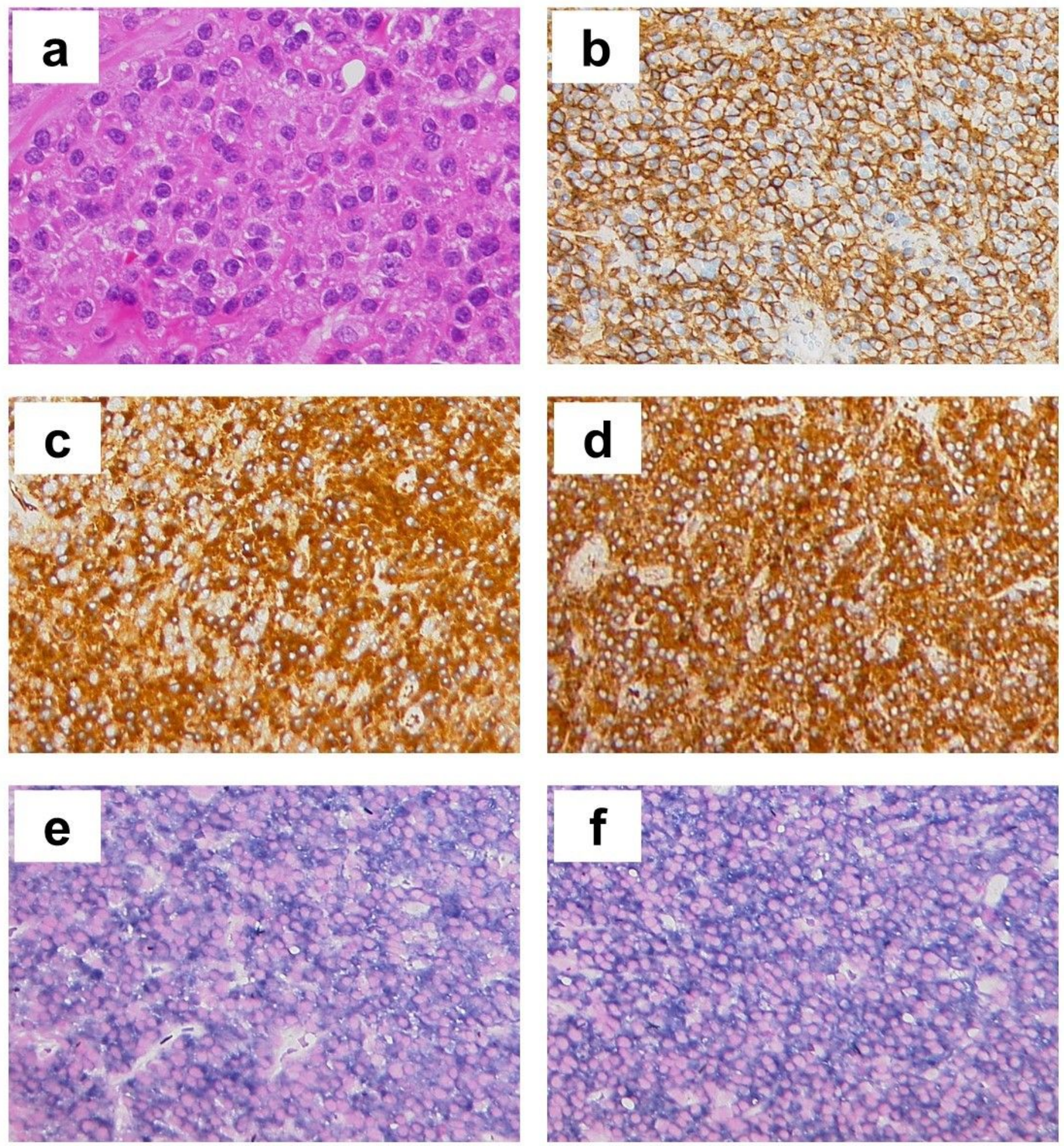

\section{Figure 3}

Tumor biopsy of the neck (sampled at 2019). (a) H\&E staining (x200) shows marked plasma cells infiltration. (b) CD138 staining (x200) shows diffuse positivity for these plasma cells. (c \& d) In situ 
hybridization of kappa and lambda light chain mRNA and (e \& f) immunohistochemistry of kappa and lambda light chain.

\section{Supplementary Files}

This is a list of supplementary files associated with this preprint. Click to download.

- 20210914CAREchecklistEnglish2013.pdf

- SupFigure1.jpg 